\title{
Analyze the Performance difference between Delay Sensitive and Delay Insensitive Routing Algorithms in Underwater Sensor Networks
}

\author{
Akanksha Dubey \\ PG Scholar, CSE Department \\ Shree Vaishnav Institute of Technology and \\ Science, Indore, India
}

\author{
Anand Rajavat \\ HOD, CSE Department \\ Shree Vaishnav Institute of Technology and \\ Science, Indore, India
}

\begin{abstract}
Under water sensor network protocol research is a hot research topic in the networking field. These protocols are designed on the basis of UWSN application requirements. Selection of protocol is depends on the basis of system requirement and its application. Real time applications are time variant and require reliability for communicating in side water. Average time to time Delay of nodes is the most important key parameter for delay-sensitive applications of UWSN. In this research researchers are working on two distributed routing protocols Delay-sensitive routing protocol (DSRP) and Delay-insensitive routing protocol (DIRP). Researchers compared the performance of the both routing solutions on the basis of some parameters; those parameters are energy consumption, Packet delivery ratio, Average endto-end delay, and Throughput. Simulation shows performance of routing algorithms for Delay-sensitive and delayinsensitive applications.
\end{abstract}

\section{General Terms}

Wireless Network, Underwater Sensor Network, Distributed Routing Algorithms.

\section{Keywords}

Underwater sensor network; DSRP; DIRP; Average end-toend Delay; Energy consumption; Packet delivery ratio; Throughput.

\section{INTRODUCTION}

A lot of research has been done in the field of earth surface communication; to cover intercontinental distance communication still sea is the best medium, nothing can replace underwater communication network hence underwater communication is evolving field for intercontinental communication. For years access to sea was limited because researchers had to use available ground level wired communication equipments but as the technology is growing Researchers have latest routing and networking equipments which can easily work inside the aquatic medium. About $70 \%$ area of the earth surface is occupied by the water so underwater sensor network can play an important role in any type of communication and transportation. Under water sensor network research work will open new doors for the marine transportation and natural ocean energy resources. Scientists can unveil many secretes of ocean life with the help of UWSN communication.

In this research paper researchers are working on two different distributed geographical algorithms of UWSN. Delaysensitive and Delay-insensitive are the application specific routing solutions provided for different applications of
UWSN. These routing solutions are designed to achieve the below objectives:

1. Better acoustic channel efficiency inside aquatic medium

2. Low packet-error rate for underwater network

Above objectives are quite contradictory but can be achieved by sending continues chain of short packets one after another without releasing the channel. Proposed algorithm allows each node to move towards its nearest hop independently with minimum use of node power to reduce energy consumption. Objectives are achieved with underwater network conditions only for static network but its distributed nature also supports the mobility. Size of each packet depends on the system requirements and available buffer size to hold the incoming packets.

In Sect. II this research will study many underwater sensor network routing algorithms on the basis of performances. Also discuss benefits and use of many Geographical routing protocols. Application specific routing solutions are discussed according to application requirements of underwater environment conditions. In Sect. III research will study underwater sensing applications, Now a day's UWSN is the favorite research field of the scientist just because of its wide range of applications. In Sect. IV research will see the performance evaluation for both the routing solutions; Performance of Delay-sensitive and Delay-insensitive routing solution depends on Energy consumption, Average end to end Delay, packet delivery ratio and throughput. In Sect. V conclusion of the research is given.

\section{RELATED WORK}

Underwater sensor networks have many additional advantages over other routing protocols which are designed to achieve specific goals and to fulfill the requirements of the underwater applications. In this scenario every routing techniques have some advantages and disadvantages over the underwater network and environmental conditions. There are many routing protocols have been implemented in the field of underwater sensor networks and these routing protocols can be classified in three types reactive, proactive and geographical routing protocols.

Proactive protocols need to have a large signaling overhead to organize the routes at the start time and same needs to be done each time whenever the network topology is changed. It happens because of changes in network topology, node failures or node mobility and has to be produced to all network devices. In this type of routing protocols scalability feature is more crucial issue so that proactive protocols not suitable for underwater sensor networks. 
Reactive protocols are good for frequently changing environment and it requires a high latency. For establishing the paths it requires source initiated route discovery process to control packets. Reactive protocols are not good for underwater sensor network because it generate high time difference between the simulation and the response to the establish the paths, which are processed by the slow propagation of acoustic signals.

Geographical routing protocols are suitable for underwater sensor network because of their scalability feature and require limited signaling. These protocols are more appropriate for localization features. By this localization perform in both 2D and 3D underwater sensor networks. Collection of data is the techniques in which number of transmissions is minimizing those are performing towards the base station by eliminating redundancy among packets from multiple sources. Geographical routing techniques require the information of the best neighbor for forwarding decision of packets in networks.

\section{Some Benefits of Geographical Routing Protocol are:}

1. Geographical routing protocol has the proficiency of Node Mobility support. In this all nodes update their routing tables according to transmission here all nodes have knowledge of its neighbor nodes information.

2. The very important property of Geographical routing is Scalability in which routing table size depends on density of network. Thus thousands of nodes containing by wider network and these can be accomplished without cluster formation.

3. In geographical routing only localized information needed, nodes should be know location of neighbors by which minimum overhead occurs. Hence transmission and execution energy consumption is less.

There are many routing protocols are proposed in the field of underwater sensor network. In [1], Many Applications are perform in underwater wireless sensor networks such as pollution monitoring, offshore exploration, disaster prevention, oceanographic data collection, assisted navigation and tactical surveillance applications. A enabling technology for these applications is Underwater acoustic networking. This network technology containing many number of sensors and AUVs acoustic underwater vehicles to perform all monitoring tasks over a given underwater area. This research provides respective fundamental key aspects of underwater acoustic communications. They discussed different architectures for 2D and 3D underwater sensor networks and also provide detailing data for the characteristics of the underwater channel. They suggested use of a cross layer approach in order to design network communication suites for efficient networking functionalities in underwater environment. Many further research work issues are also discussed with possible solution approaches in underwater sensor networks.

In [2], Routing function of the network layer provides the communication path to the nodes in the network, in this algorithms are provide according to the application requirements of the underwater sensor network. Applications such as pollution monitoring, disaster prevention, tactical surveillance, oceanographic data collection and many more. According to underwater environment architecture for 3D underwater sensor network introduced in this paper. In this research two distributed routing algorithms are investigated for different applications which are: Delay-sensitive and Delay-insensitive applications. To achieve the objective of minimizing the energy consumption according to the different application requirements and variable conditions of the underwater channel.

In [3], Underwater Acoustic sensor network mainly works on sensing and processing capabilities of nodes to perform collaborative monitoring tasks of different applications. There is communication technology which supports communication over one hundred meters distance in wireless acoustic sensor networking. The idea operates on account of large attenuation as well as scattering which affect radio stations as well as optical waves. In this research two geographical routing algorithms are introduced on the basis of underwater applications. Those are Delay-sensitive applications and Delay-insensitive applications. The main objective of these algorithms is to minimize the energy consumption in varying conditions of underwater acoustic channel and also in different applications scenario.

In [4], underwater sensor network and latest multimedia technology together can open a new chapter of underwater multimedia acoustic sensor network which can be used in wide range of applications like disaster perdition and prevention, coastal surveillance, under ocean explorations, oceanographic research work and seismic decay activity. The main goal of this research is to analyze the underwater communication functionalities on the basis of transmission are modulation, medium access control and routing. There is a lot of variation in the applications so it required to provide a very accurate and efficient service support to delay tolerant and loss tolerant as well as delay sensitive and delay tolerant traffic.

In [5], this research provides A Survey on different routing algorithms in underwater sensor networks. In underwater the sensor nodes placed at the seafloor, and it require multi-hop communication which provided by any selective routing schemes. These routing schemes selection depends on application requirements, network resources and also on environmental constraints. On the basis of different application requirements with dynamic environment conditions routing schemes were design. Many routing protocols have been implemented to provide efficient communication path between source and the sink. Review for different routing protocols provided in order to complete above requirements are present in this paper. The main focus of this review study is to present the certain issues such as localization, data forwarding and deployment of the sensor nodes in UWSNs on the basis of different conditions. Also these routing schemes are classified according to their functionalities and characteristics into different groups.

In [6], Localization is the important key factor in the field of underwater communication, it define as the determining the location of each node in a underwater sensor network. There are some localization algorithms are investigated for underwater sensor network, these are different from localization algorithms of terrestrial network in some characteristics. In this research they describe some localization algorithms for underwater sensor network, and also shows challenges which are required at the time of application processing scenario. 
In [7], Many applications are perform in underwater sensor network field. In this particular research these people identified the facts gathering difficulty in 3D underwater sensor network by interactions between routing features and the qualities of the underwater channel in the network level associated with transmission technology. To own objective involving survivability on the network to node in addition to link failure problems, a Two-phase routing protocol is implemented. This routing algorithm implemented for longterm monitoring applications of underwater sensor network. In this energy efficient paths are configured by relaying on network topology information gathered by a source station, and these paths are repaired locally when node failures occur.

In [8], underwater inter-vehicle communication capabilities are used by many geographical applications for communication or information exchange in underwater sensor networks. Reliability is the main concern in underwater field for data delivery; all applications required this factor in communication process. This research proposed three versions of protocol for reliable communication. Protocol used different levels of neighbor knowledge to taking optimum decision. There are three different levels of neighbor knowledge are 1. No neighbor knowledge 2.one-hop neighbor knowledge 3. Two-hop neighbor knowledge. The comparison performed on static as well as mobile scenarios on the basis of some different networking metrics such as: packet delivery ratio, packet delay and energy consumption. In [9], underwater wireless sensor network protocols performance can major by many parameters but energy efficiency is the very essential parameter which consider for the selection of the routing protocol in underwater environment. Underwater communication technologies affected by some parameters such as: networking dynamics, high error probability and large propagation delays. This research analyzes the total energy consumption for routing algorithms on the basis of two different scenarios which are Shallow water and Deep water. They proposed basic functioning principles: relaying, direst transmission and clustering for routing protocols in underwater acoustic networks. Also analyze the energy consumption in both the scenarios and provide comparison mechanism between them.

In [10], In this research work will have performed on different applications of underwater sensor network. Main applications on which research done are seismic monitoring for oil extractions from underwater, equipment monitoring, underwater robotics and leak detection. This research described the directions of research in the area of short-range acoustic communications, MAC time synchronization, localization protocols for acoustic networks and applicationlevel data scheduling. It provides review to the different characteristics of all these applications.

In [11], In this research Hierarchical and cluster based Routing protocol designed for wireless sensor network. It provides mobility in the sensor nodes and also in the sink. There are two phase in protocol setup phase and data forwarding phase. In this process sensor field divided into some logical clusters. Every cluster contains different types of node such as Cluster head nodes, Gateway nodes and ordinary sensor node. Research shows the performance of the proposed protocol on the basis of the energy efficiency and also compares it with the CBR mobile WSN protocol and show better performance than CBR.
In [12], this paper analyzes the many routing protocols in the field of underwater sensor networks. It presents a classification for the many approaches. This paper represents the three categories such as: location based, hierarchical and data centric. Presented routing protocols are explored and discussed under the suitable category in the network. Protocols are also using some methodologies those are quality of service and network flow on these discussion provided by authors.

In [13], Researchers provide a lot of analysis or survey of routing protocols in wireless sensor network depends on application requirements. Different applications required different routing solutions so that a routing algorithm is working for all applications is not possible. This research provides the five categories for different applications and common routing protocols of wireless networks are define, classified and studied.

\section{UNDERWATER SENSING APPLICATIONS}

some decades ago underwater sensor network was a hidden field for the researchers but as the technology grew up many new research happened in the field of underwater instrumentation which opened new doors of research in the field of UWSN because the major limitation was lack of the equipment which can perform accurately into the water. Now a day's UWSN is hot research field of the scientist just because of its wide range of applications. Application domain of USWN varies with the same factors which affect the terrestrial sensor network like temperature of water, its bacterial content, ph value etc. Applications of USWN are:

\subsection{Eye on underwater life}

Since current era is the era of science and technology which bring two different side out of that one which show us the way of development and the other one is disturbance in nature, like when people establish a firm \& they deploy its waste part in the water which causes imbalance in underwater life. We can save the underwater life by showing the research work to the world that how badly the underwater life is disturbed by their activity.

\subsection{Seismic monitoring}

One of the best application of UWSN is seismic monitoring for gas and oil extraction from the beneath of the earth. Since ocean is very dynamic so it needs continuous time based observation of all the affecting components. 4-D seismic observation is very useful for judging and observing field performance. Monitoring of interstitial oil sources are quite easy because it don't require continuous monitoring unlike continuous monitoring of underwater oil reservoirs. Monitoring of underwater oil reservoir is not so easy because the underwater sensors are not permanently placed inside the ocean. Seismic monitoring is done with the help of sensor dipped inside ocean with the help of a ship which send the observation data of underwater oil field through air cannons..

\section{PERformance eValuation}

This research work is representing the simulation performance of the application specific routing solutions for Delaysensitive and Delay-insensitive in underwater sensor network. Research work compared the performance of both routing solutions on the basis of some parameters: Packet delivery ratio, average end-to-end delay, energy consumption and 
throughput. Simulation shows performance of routing algorithms for Delay-sensitive and delay-insensitive applications. Researchers are working on Aqua-sim simulator for analyzing the performance of both routing algorithms.

\subsection{Energy consumption performance for DIRP \& DSRP}

This research observes the energy consumption parameter of delay-sensitive routing protocol (DSRP) and delay-insensitive routing protocol (DIRP). Here energy consumption unit is energy consumed*10^3. Graph represents the Number of node vs. energy consumption performance of both the routing protocols. Observation drawn by graph is that in the path of transmission on a particular point DIRP has larger energy consumption than DSRP and again both routing having same energy consumption. Energy consumed by both the algorithms are quite similar in increasing manner but when number of nodes are greater, than energy consumption by both protocols are increased. Research also observed that delay-sensitive routing protocol (DSRP) energy consumption performance is more than delay-insensitive routing protocol (DIRP).

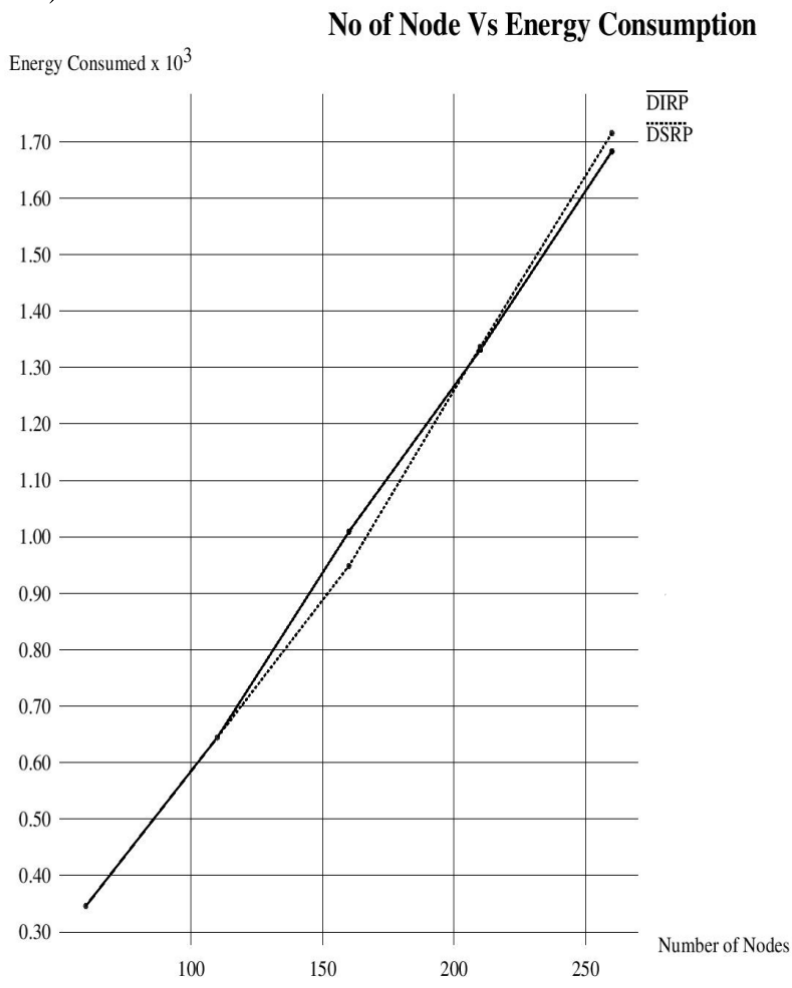

Fig 1: Number of nodes vs. Energy consumption graph for DSRP \& DIRP routing protocol

\subsection{Average End to End Delay performance for DIRP \& DSRP.}

This research observes the Average end-to-end Delay parameter of delay-sensitive routing protocol (DSRP) and delay-insensitive routing protocol (DIRP). Here Delay takes in time and unit is ms (millisecond). Graph represents the Number of node vs. time (ms) performance of both the routing protocols. Observation drawn by graph is that in the path of transmission on a particular point DIRP and DSRP Avg. end to end delay are same. Now in further transmission DIRP has greater Avg. end to end delay than DSRP routing protocol. Average end to end Delay of both the algorithms are quite similar in increasing manner but when number of nodes are greater, than Avg. end to end Delay by both protocols are increased. Research also observed that delay-insensitive routing protocol (DIRP) Avg. end to end Delay performance is more than delay-sensitive routing protocol (DSRP).

Avg. end to end delay (ms)

time

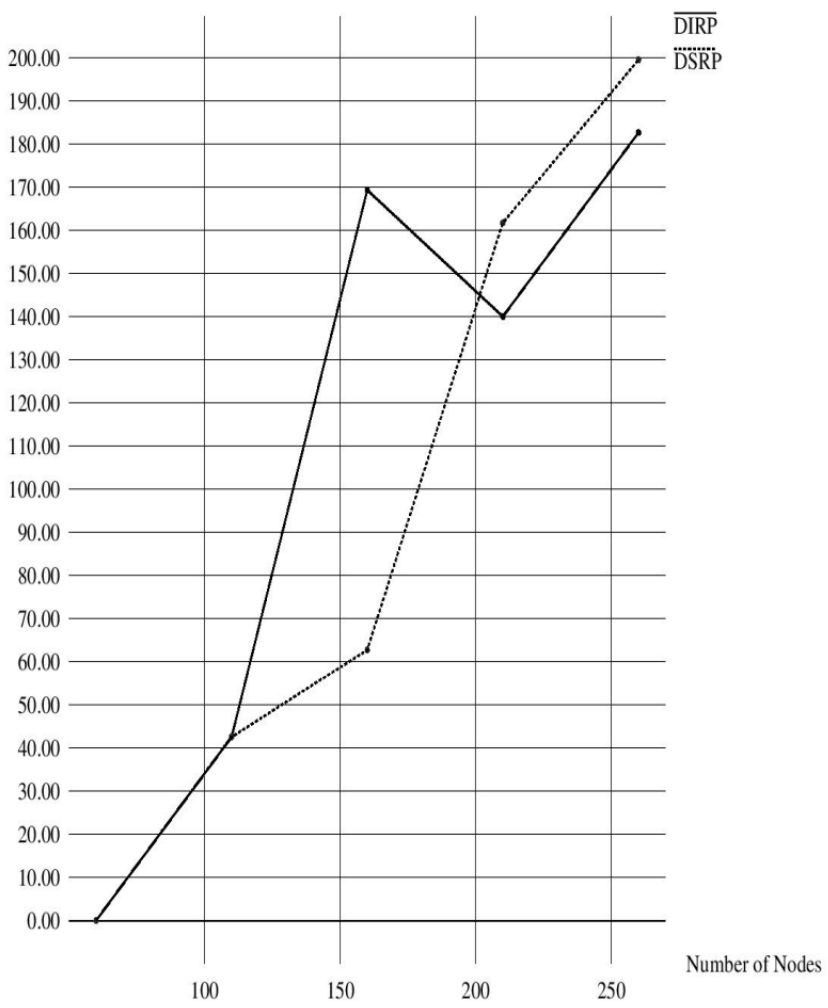

Fig 2: Number of nodes vs. Avg. end to end Delay graph for DSRP \& DIRP routing protocol

\subsection{Packet delivery ratio performance for DIRP \& DSRP.}

This research observes the packet delivery ratio parameter of delay-sensitive routing protocol (DSRP) and delay-insensitive routing protocol (DIRP). Here packet delivery ratio unit is $\operatorname{PDR} * 10^{\wedge}-3$. Graph represents the Number of node vs. Packet delivery ratio performance of both the routing protocols. Observation drawn by graph is that in the path of transmission on a particular point DIRP has larger packet delivery ratio than DSRP and now in further transmission DIRP has less packet delivery ratio than DSRP routing protocol. Packet delivery ratio of both the algorithms are quite similar in increasing manner but when number of nodes are greater, than packet delivery ratio by both protocols are increased. Research also observed that delay-sensitive routing protocol (DSRP) packet delivery ratio performance is more than delay-insensitive routing protocol (DIRP). 
Packet Delivery Ratio

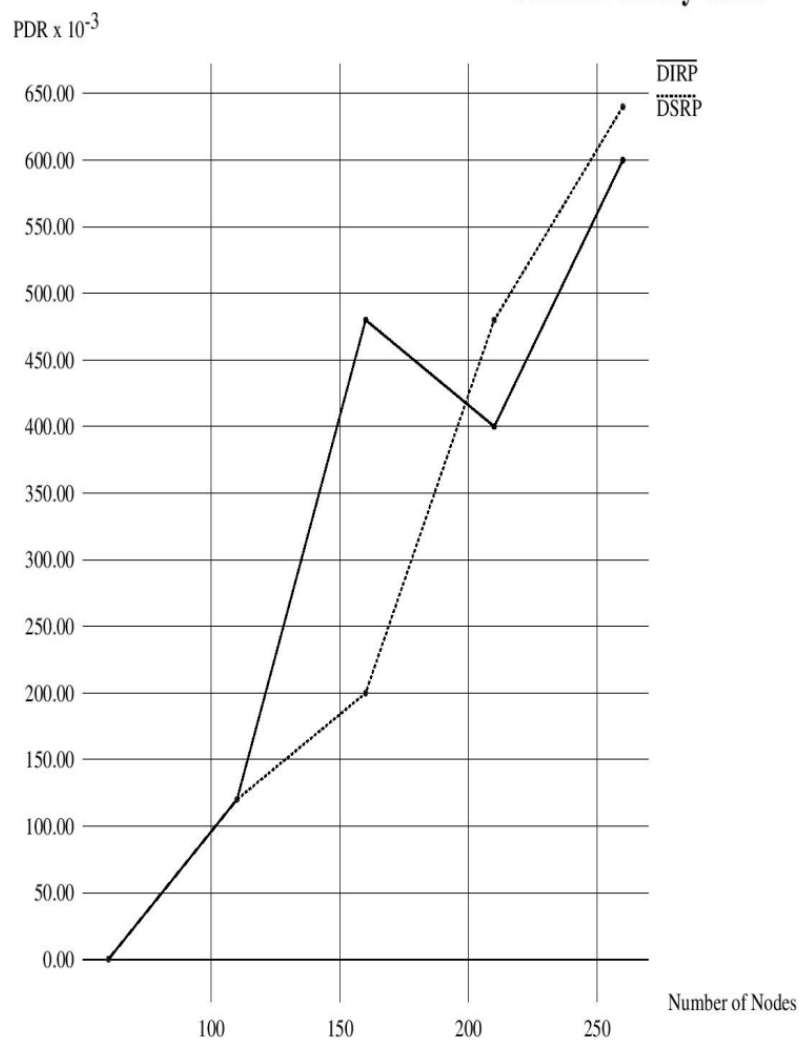

Fig 3: Number of nodes vs. Packet Delivery Ratio graph for DSRP \& DIRP routing protocol

\subsection{Throughput performance for DIRP \& DSRP}

This research observes the throughput parameter of delaysensitive routing protocol (DSRP) and delay-insensitive routing protocol (DIRP). Here throughput measure in $\mathrm{KB} / \mathrm{Second}$. Graph represents the Number of nodes vs. throughput performance of both the routing protocols. Observation drawn by graph is that in the path of transmission on a particular point both DIRP and DSRP has same throughput, now in further transmission on same number of nodes DIRP has more throughput than DSRP. Performance of throughput of both the algorithms is quite similar in increasing manner but when numbers of nodes are greater, than throughput of both the protocols is increased. Research also observed that delay-sensitive routing protocol (DSRP) throughput performance is more than delay-insensitive routing protocol (DIRP).
Throughput (KB/s)

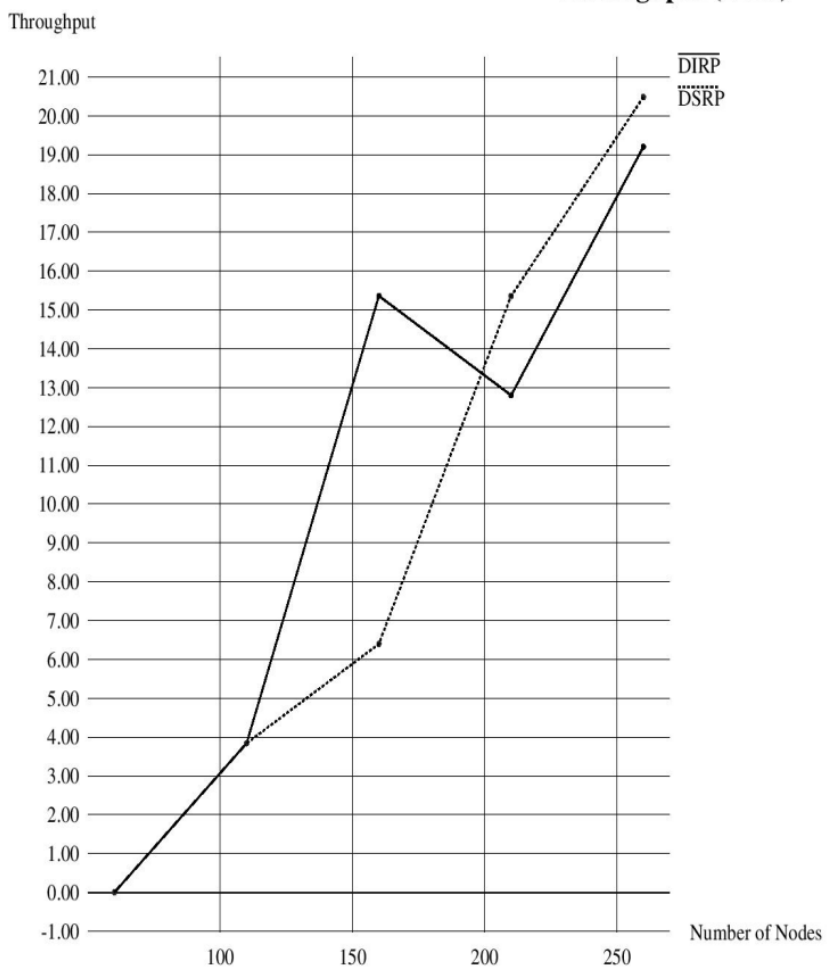

Fig 4: Number of nodes vs. Throughput graph for DSRP \& DIRP routing protocol

\section{CONCLUSION}

This research provides evaluation of two distributed geographical routing protocols Delay-sensitive routing protocol (DSRP) and Delay-insensitive routing protocol (DIRP) through Aqua-sim simulator. These are application specific routing protocols working on delay-sensitive and delay-insensitive applications of underwater sensor network. Research performed the comparison between the performances of the both routing solutions on the basis of some parameters: energy consumption, Packet delivery ratio, Average end-to-end delay, and Throughput.

Future work involves node mobility concept and their effects on energy efficiency issue of both distributed routing delaysensitive and delay- insensitive algorithms in underwater sensor network. This node mobility concept will be performing in sparse and dense network of AUVs on the basis of parameters which are discussed above.

\section{REFERENCES}

[1] I. F. Akyildiz, D. Pompili, and T. Melodia, "Underwater acoustic sensor networks: research challenges," Ad Hoc Networks, vol. 3, no. 3, pp.257-279, 2005

[2] D. Pompili, T. Melodia, and I. F. Akyildiz, "Routing algorithms for delay-insensitive and delay-sensitive applications in underwater sensor networks," in Proc. ACM Conf. Mobile Comput. Netw. (MobiCom),Los Angeles, CA, Sep. 2006.

[3] Distributed Routing Algorithms for Underwater Acoustic Sensor Networks Dario Pompili, Member, IEEE, Tommaso Melodia, and Ian F. Akyildiz, IEEE transactions on wireless communications, vol. 9, no. 9, september 2010. 
[4] D. Pompili and I. F. Akyildiz, "A cross-layer communication solution for multimedia applications in underwater acoustic sensor networks," in Proc. IEEE International Conf. Mobile Ad-hoc Sensor Syst. (MASS), Atlanta, GA, Sep. 2008.

[5] Muhammad Ayaz n, Imran Baig, Azween Abdullah, Ibrahima Faye "A survey on routing techniques in underwater wireless sensor networks" CIS Department, EE Department, FAS Department, Universiti Teknologi PETRONAS, Malaysia Journal of Network and Computer Applications Article Elsevier 2011.

[6] V. Chandrasekhar, W. K. Seah, Y. S. Choo, and H. V. Ee, "Localization in underwater sensor networkssurvey and challenges," in Proc. ACM International Workshop UnderWater Netw. (WUWNet), Los Angeles, CA, Sep. 2006.

[7] D. Pompili, T. Melodia, and I. F. Akyildiz, "A resilient routing algorithm for long-term applications in underwater sensor networks," in Proc. Mediterranean Ad Hoc Netw. Workshop (Med-Hoc-Net), Lipari, Italy, June 2006.

[8] A. Nimbalkar and D. Pompili, "Reliability in underwater inter-vehicle communications," in Proc. ACM International Workshop UnderWater Netw. (WUWNet), San Francisco, CA, Sep. 2008.

[9] Mari Carmen Domingo, Rui Prior, "Energy analysis of routing protocols for underwater wireless sensor networks" in ScienceDirect computer communication Article 31 Elesvier, 2008, pp. 1227-1238.

[10] John Heidemann Milica Stojanovic and Michele Zorzi, "Underwater sensor networks: applications, advances and challenges" in Phil. Trans. R. Soc. A, 3702012 pp.158175 .
[11] Sarma, H.K.D. Kar, A. Mall, R. "Energy efficient routing protocol for Wireless Sensor Networks with Node and Sink mobility" in Sensors Applications Symposium (SAS), 2011 IEEE Date 22-24 Feb. 2011 San Antonio, TX.

[12] K. Akkaya and M. Younis, "A survey on routing protocols for wireless sensor networks," Ad Hoc Netw. (Elsevier), vol. 3, no. 3, pp. 325-349, May 2005.

[13] Long Zhaohua, ChongQing, Liu Jianfeng; Jiang Guiquan "Survey of routing protocols in wireless sensor networks based on applications" in 2nd International Conference on Industrial and Information Systems (IIS), in 2010 .

[14] M. Zorzi, P. Casari, N. Baldo, and A. Harris, "EnergyEfficient Routing Schemes for Underwater Acoustic Networks," Selected Area in Communications, IEEE Journal on, vol. 26, no. 9, pp. 1754-1766, 2008 [Online]. Available: http://dx.doi.org/10.1109/JSAC.2008.081214

[15] P. Xie, J.-H. Cui, and L. Lao, "VBF: vector-based forwarding protocol for underwater sensor networks," in Proc. Netw., Coimbra, Portugal, May 2006.

[16] W. Zhang and U. Mitra, "A delay-reliability analysis for multihop underwater acoustic communication," in Proc. ACM International Workshop UnderWater Netw. (WUWNet), Montreal, Quebec, Canada, Sep. 2007.

[17] P. Xie, Z. Zhou, Z. Peng, H. Yan, T. Hu, J.-H. Cui, Z Shi, Y. Fei, and S. Zhou, "Aqua-sim: An ns-2 based simulator for underwater sensor networks," in OCEANS 2009, MTS/IEEE Biloxi - Marine Technology for Our Future: Global and Local Challenges, 2009, pp. 1-7. 